\title{
A functional interaction of SmpB with tmRNA for determination of the resuming point of trans-translation
}

\author{
TAKAYUKI KONNO, ${ }^{1,2,3}$ DAISUKE KURITA, ${ }^{1,2}$ KAZUMA TAKADA, ${ }^{1,2}$ AKIRA MUTO,,${ }^{1,2,4}$ \\ and HYOUTA HIMENO ${ }^{1,2,4}$ \\ ${ }^{1}$ Department of Biochemistry and Biotechnology, Faculty of Agriculture and Life Science, Hirosaki University, Hirosaki 036-8561, Japan \\ ${ }^{2}$ The United Graduate School of Agricultural Sciences, Iwate University, Morioka 020-8550, Japan \\ ${ }^{3}$ Department of Microbiology, AKITA Prefectural Research Center for Public Health and Environment, Akita 010-0874, Japan \\ ${ }^{4}$ RNA Research Center, Hirosaki University, Hirosaki 036-8561, Japan
}

\begin{abstract}
In trans-translation, transfer-messenger RNA (tmRNA), possessing a dual function as a tRNA and an mRNA, relieves a stalled translation on the ribosome with the help of SmpB. Here, we established an in vitro system using Escherichia coli translation and trans-translation factors to evaluate two steps of trans-translation, peptidyl transfer from peptidyl-tRNA to alanyl-tmRNA and translation of the resume codon on tmRNA. Using this system, the effects of several mutations upstream of the tag-encoding region on tmRNA were examined. These mutations affected translation of the resume codon rather than peptidyl transfer, and one of them, A84U/U85G, caused a shift of the resume codon by -1 . We also found that $U_{85}$ is protected from chemical modification by SmpB. In the A84U/U85G mutant, the base of protection was shifted from 85 to 84 . Another mutation, A86U, which caused a shift of the resume codon by +1 , shifted the base of protection from 85 to 86 . The protection at 85 was suppressed by a mutation in the tRNA-like domain critical to SmpB binding. These results suggest that SmpB serves to bridge two separate domains of tmRNA to determine the initial codon for tag-translation. A mutant SmpB with a truncation of the unstructured C-terminal tail failed to promote peptidyl transfer, although it still protected $U_{85}$ from chemical modification.
\end{abstract}

Keywords: tmRNA; trans-translation; SmpB; chemical modification

\section{INTRODUCTION}

Transfer-messenger RNA (tmRNA), also known as 10Sa RNA or SsrA RNA, having both tRNA and mRNA properties, is widely distributed among eubacteria and has also been found in some chloroplasts and mitochondria (Gueneau de Novoa and Williams 2004; Andersen et al. 2006). Both ends of tmRNA fold into a tRNA-like structure (Williams and Bartel 1996; Felden et al. 1997), allowing its 3 '-end to be aminoacylated with alanine (Komine et al. 1994; Ushida et al. 1994), followed by binding to EF-Tu (Rudinger-Thirion et al. 1999; Barends et al. 2000; Stepanov and Nyborg 2003) and subsequently to the ribosome stalled on a truncated mRNA lacking a stop codon (Keiler et al. 1996; Muto et al. 1998) to receive a nascent polypeptide from peptidyl-tRNA at the P-site. This noncanonical trans-

Reprints requests to: Hyouta Hiemno, Department of Biochemistry and Biotechnology, Faculty of Agriculture and Life Science, Hirosaki University, Hirosaki 036-8561, Japan; e-mail: himeno@cc.hirosaki-u.ac.jp; fax: 81-172-39-3593.

Article published online ahead of print. Article and publication date are at http://www.rnajournal.org/cgi/doi/10.1261/rna.604907. lation, called trans-translation, produces a chimera peptide fusing the tmRNA-encoded tag-peptide at the $\mathrm{C}$ terminus as a degradation signal (Keiler et al. 1996) and allows the ribosome to be recycled back to a new round of translation for various kinds of cellular events (Abo et al. 2000; Huang et al. 2000; Muto et al. 2000; Fujihara et al. 2002; Keiler and Shapiro 2003; Withey and Friedman 2003).

Since it is assumed that there is no apparent codonanticodon interaction before the first translocation event of trans-translation, the mechanism by which tmRNA resumes translation from the first GCA codon for a tag-peptide is a mystery. It should require an elaborate coordination between the two functional domains apparently distant from each other on the secondary structure (Hanawa-Suetsugu et al. 2001). The first pseudoknot (PK1) 12 nucleotides (nt) upstream of the tag-initiation point, but not the other three pseudoknots (PK2-PK4) downstream from the tag-encoding region, has been shown to be important for efficient tagtranslation (Nameki et al. 1999a, b, 2000). It has also been shown both in vivo (Williams et al. 1999) and in vitro (Lee et al. 2001) that 2-5 nt upstream of the tag-encoding region are involved in efficient tag-translation. Interestingly, some 
base substitutions around this region cause an apparent incorporation of amino acids comprising the -1 frame tagpeptide in vitro (Lee et al. 2001). Thus, the upstream sequence on tmRNA might have an intermolecular interaction with the stalled ribosome to serve as a determinant for the initiation point of tag-translation, although whether it occurs directly or via a trans-acting factor and how it dynamically rearranges during the course of the transtranslation processes remain unsolved.

EF-Tu, SmpB (Barends et al. 2001; Karzai et al. 1999; Hanawa-Suetsugu et al. 2002), and ribosomal protein S1 (Wower et al. 2000) have been identified as tmRNAbinding proteins. Among them, SmpB is thought to be a key molecule essential for trans-translation. It binds the tRNA-like domain (TLD) to perform multiple functions in the processes of trans-translation, recruitment of tmRNA to the stalled ribosome (Karzai et al. 1999; Hanawa-Suetsugu et al. 2002), enhancement of aminoacylation of tmRNA by alanyl-tRNA synthetase (Barends et al. 2001; HanawaSuetsugu et al. 2002; Shimizu and Ueda 2002), enhancement of processing of tmRNA (Wower et al. 2004), and protection of tmRNA from degradation in the cell (Hanawa-Suetsugu et al. 2002; Hong et al. 2005). NMR studies have revealed that $\mathrm{SmpB}$ is comprised of an antiparallel $\beta$-barrel core with three $\alpha$-helices and C-terminal basic residues that are disordered in solution (Dong et al. 2002; Someya et al. 2003). In a crystal structure of $S m p B$ in complex with a fragment of the tRNA-like domain (TLD) (Gutmann et al. 2003), SmpB binds the elbow of TLD, in agreement with results of biochemical studies (Hanawa-Suetsugu et al. 2002; Nameki et al. 2005), and $\mathrm{SmpB}$ can be superimposed on the anticodon stem and loop of tRNA, if TLD is fixed on the amino acid acceptor stem and the T-arm, implying that SmpB functions around the decoding region during trans-translation. Although with no structural information about the C-terminal region, it has recently been shown that $\mathrm{SmpB}$ having a mutation or truncation in the C-terminal tail does not facilitate trans-translation in vivo (Jacob et al. 2005; Sundermeier et al. 2005). This raises the possibility that the C-terminal tail introduces the first GCA codon of tmRNA to the A-site of the 70S ribosome by interacting with some part of tmRNA such as the upstream sequence in proximity to the decoding center of the ribosome.

In the present study, we established an in vitro system using Escherichia coli factors specific for translation and trans-translation to evaluate two steps of trans-translation, peptidyl transfer from peptidyl-tRNA to alanyl-tmRNA and translation of the resume codon on tmRNA. Using this system, we identified the effects of mutations introduced in the upstream sequence of the tag-encoding region on tmRNA, which include the loss of efficiency or specificity of translation of the resume codon. We also found that the uridine residue $5 \mathrm{nt}$ upstream of the tag-initiation point is significantly protected from chemical modification by SmpB. A mutation introduced in the upstream sequence of the tag-encoding region shifted the position of protection apparently in relation to the shift of the initiation point. Finally, we found that the protection was not due to interaction with the C-terminal tail.

\section{RESULTS}

\section{In vitro trans-translation system to evaluate peptidyl transfer to alanyl-tmRNA and translation of the resume codon on tmRNA}

Several in vitro trans-translation systems have been developed to evaluate tag-peptide synthesis using cell extracts from E. coli (Himeno et al. 1997) or peptidyl transfer from peptidyl-tRNA to alanyl-tmRNA (the first step) using translation and trans-translation factors from E. coli (Shimizu and Ueda 2002; Asano et al. 2005). In the present study, we used the system of Asano et al. (2005) and extended it to an in vitro system to evaluate two steps of trans-translation, not only the first step but also translation of the resume codon on tmRNA (the second step).

First, polyphenylalanine was synthesized from (UUC) 10 using ribosome, phenylalanyl-tRNA ${ }^{\text {Phe }}$, and elongation factors to produce a stalled complex of ribosome (Asano et al. 2005). After $15 \mathrm{~min}$ of incubation, $\sim 1 \mathrm{pmol}$ of $\left[{ }^{14} \mathrm{C}\right]$ phenylalanine was incorporated into $\left[{ }^{14} \mathrm{C}\right]$ polyphenylalanyl-tRNA $^{\text {Phe }}$. This stalled complex of ribosome with $\left[{ }^{14} \mathrm{C}\right]$ polyphenylalanyl-tRNA ${ }^{\text {Phe }}$ at the P-site was then incubated with ${ }^{3} \mathrm{H}$-labeled alanyl-tmRNA to evaluate the first step. Incorporation of $\sim 190 \mathrm{fmol}$ of $\left[{ }^{3} \mathrm{H}\right]$ alanine into the polypeptide fraction designating the formation of $\left[{ }^{14} \mathrm{C}\right]$ polyphenylalanyl- $\left[{ }^{3} \mathrm{H}\right]$ alanyl-tmRNA was observed after $5 \mathrm{~min}$ of incubation only in the presence of $\mathrm{SmpB}$ (Fig. 1A). To evaluate the second step, the stalled complex of ribosome was incubated with unlabeled alanyl-tmRNA, SmpB, and any one of $\left[{ }^{3} \mathrm{H}\right]$ alanyl-tRNA ${ }^{\text {Ala }},\left[{ }^{3} \mathrm{H}\right]$ arginyltRNA ${ }^{\mathrm{Arg}}$, and $\left[{ }^{3} \mathrm{H}\right]$ glutaminyl-tRNA ${ }^{\mathrm{Gln}}$ (corresponding to the $\mathrm{G}_{90} \mathrm{C}_{91} \mathrm{~A}_{92}, \mathrm{C}_{89} \mathrm{G}_{90} \mathrm{C}_{91}$, and $\mathrm{C}_{91} \mathrm{~A}_{92} \mathrm{~A}_{93}$ codons, respectively). Substantially, only $\left[{ }^{3} \mathrm{H}\right]$ alanine incorporation designating the formation of $\left[{ }^{14} \mathrm{C}\right]$ polyphenylalanyl-alanyl$\left[{ }^{3} \mathrm{H}\right]$ alanyl-tRNA ${ }^{\mathrm{Ala}}$ was observed (Fig. 1B), indicating that the present in vitro tag-peptide translation was actually initiated almost exclusively from the GCA codon at 90-92 as suggested by previous in vivo (Keiler et al. 1996) and in vitro studies (Himeno et al. 1997; Lee et al. 2001).

\section{Effects of mutations upstream of the tag-encoding sequence of tmRNA on the resumption of trans-translation}

Lee et al. (2001) showed by using E. coli cell extract that mutations in the upstream sequence of the tag-encoding region of tmRNA significantly affect poly(U)-dependent tagpeptide synthesis in vitro. In the present study, we selected 
A

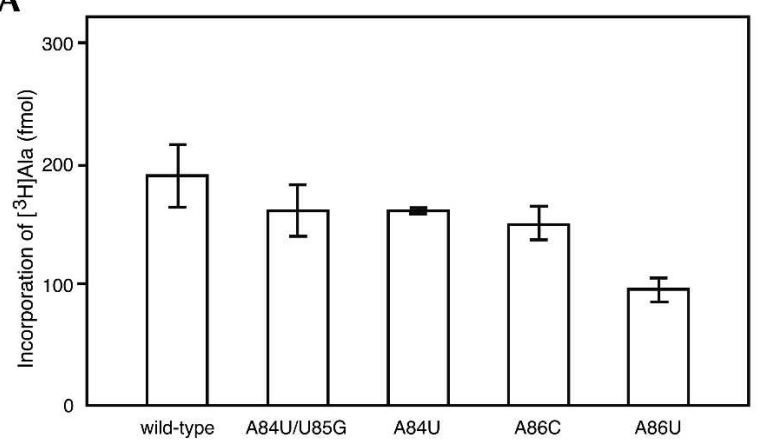

B

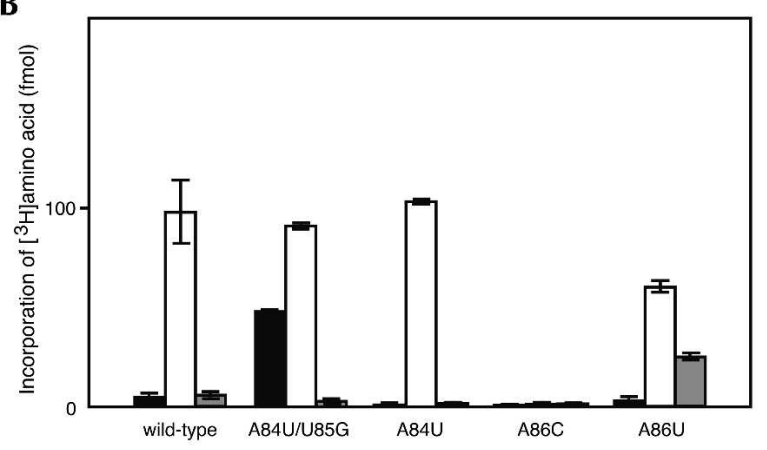

C

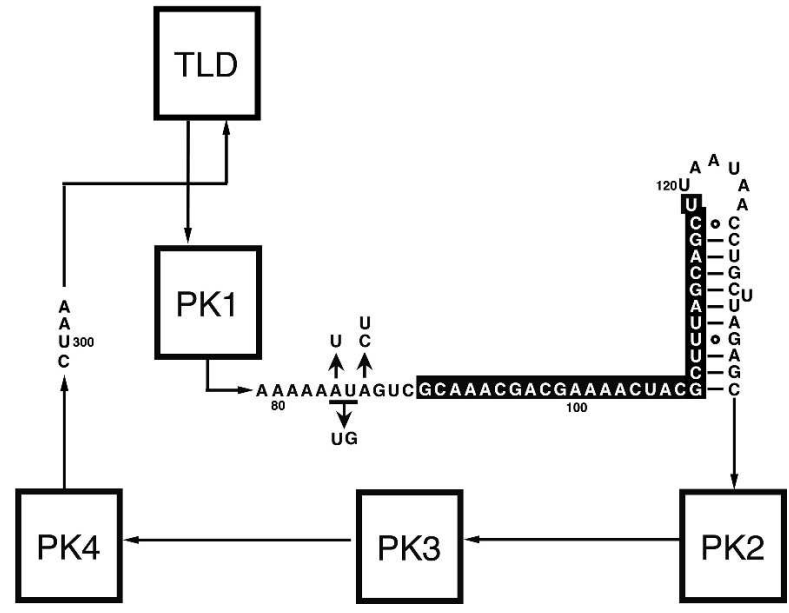

FIGURE 1. The first and second steps of trans-translation facilitated by tmRNA variant and SmpB. (A) The level of incorporation of $\left[{ }^{3} \mathrm{H}\right]$ alanine into the polypeptide fraction after $5 \mathrm{~min}$ of incubation was monitored to evaluate the first step of trans-translation. (B) The levels of incorporation of $\left[{ }^{3} \mathrm{H}\right]$ arginine (black), $\left[{ }^{3} \mathrm{H}\right]$ alanine (white), and $\left[{ }^{3} \mathrm{H}\right]$ glutamine (gray) into the polypeptide fraction after $5 \mathrm{~min}$ of incubation were monitored to evaluate the efficiency and fidelity of the second step of trans-translation. $(C)$ Mutations used in the present study are designated on the secondary structure model of tmRNA, where TLD, PK1, and pseudoknots PK1-4 are abbreviated in boxes, and the tag-encoded sequence is in white letters on a black background.

three typical mutations, A84U/U85G, A84U, and A86C, that cause a -1 frameshift of tag-translation up to $\sim 60 \%$, almost no damage, and a serious decrease in the efficiency of tagtranslation, respectively (Fig. 1C), to examine the effects on both the first and second steps of trans-translation using the system that we developed as described above. The levels of alanine incorporation for all of these mutants in the first step were comparable to that of the wild type (Fig. 1A). The pattern of amino acid incorporation in the second step for the A84U mutant was similar to that for the wild type (Fig. $1 B)$. When the A84U/U85G mutant was used, arginine was considerably incorporated in the second step, although glutamine was not (Fig. 1B). The level of incorporation of arginine was about half of that of alanine. This result indicated that the start position of the tag-translation was significantly shifted by -1 . This proved that an apparent incorporation of amino acids comprising the -1 frame tagpeptide observed in a previous study (Lee et al. 2001) was actually due to the initiation shift of tag-translation. When the A86C mutant was used, there was little incorporation of any of the three amino acids in the second step (Fig. 1B), which is consistent with the results of a previous study using E. coli cell extracts (Lee et al. 2001).

We also examined the effect of the A86U mutation on the present in vitro trans-translation system. The effect of this mutation is rather controversial. No tag has been detected at the $\mathrm{C}$ terminus of a reporter peptide in vivo (Williams et al. 1999), while tag-translation with $50 \%-60 \%$ efficiency has been detected in vitro using E. coli cell extracts (Lee et al. 2001). The efficiencies of alanine incorporation at the first and second steps were $\sim 50 \%$ and $50 \%$, respectively (Fig. $1 \mathrm{~A}, \mathrm{~B})$. The results are consistent with a previous in vitro study using cell extracts. We also detected glutamine incorporation at the second step designating $\mathrm{a}+1$ initiation shift (Fig. 1B). The efficiency of glutamine incorporation was $\sim 40 \%$ of that of alanine incorporation. A low level of +1 shift has also been detected in a previous in vitro study using cell extracts (Lee et al. 2001).

\section{Interaction of SmpB with the upstream region of the tag-encoding sequence of tmRNA}

We performed a chemical footprinting study using DMS, $\mathrm{KE}$, and CMCT to examine the interaction of SmpB with the upstream region of the tag-encoding sequence of tmRNA. DMS methylates $\mathrm{A}$ at position $\mathrm{N} 1$ and $\mathrm{C}$ at position $\mathrm{N} 3$ to a lesser extent, KE modifies $\mathrm{G}$ at position $\mathrm{N} 1$ and $\mathrm{N} 2$, and CMCT modifies $\mathrm{U}$ at position $\mathrm{N} 3$ and $\mathrm{G}$ at position $\mathrm{N} 1$ to a lesser extent. Chemical modification of tmRNA in the presence and absence of $6 \mu \mathrm{M}$ SmpB was performed, and the extents of chemical modification at each position were compared (Fig. 2). In the absence of $\mathrm{SmpB}$, the band of $\mathrm{U}_{85}$ was clearly observed, whereas the band of $\mathrm{U}_{88}$ was not, probably reflecting the conformation around this region as shown previously (Felden et al. 1997). It was found that the band intensity of $\mathrm{U}_{85}, 5 \mathrm{nt}$ upstream of the tag-initiation point, was decreased by the addition of $\mathrm{SmpB}$, indicating the protection of $\mathrm{U}_{85}$ from CMCT modification by $\mathrm{SmpB}$. Interaction of $\mathrm{SmpB}$ with the region between PK1 and the tag-encoding sequence has 


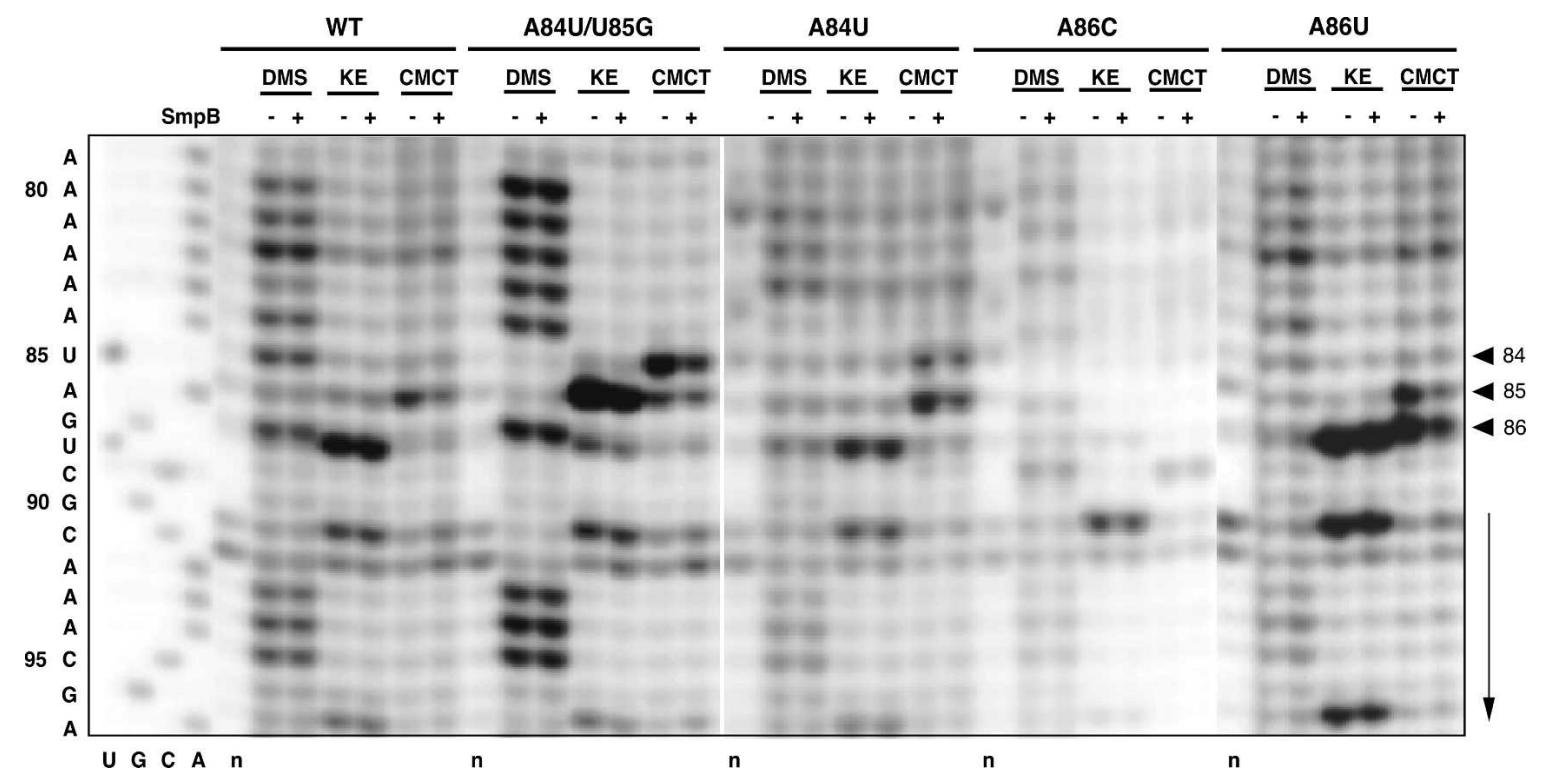

FIGURE 2. Chemical footprinting of tmRNA by SmpB. Wild-type tmRNA, A84U/U85G, A84U, and A86C were modified with DMS, KE, and $\mathrm{CMCT}$ in the presence and absence of $6 \mu \mathrm{M}$ SmpB. The modified tmRNA variants were analyzed by primer extension. The band appears at the site of interruption of reverse transcription just before the base of modification. (Arrowheads) The bands of interest; (arrow) the tag-encoding region with the direction. The lanes of DNA sequencing of pGEMEX-2 carrying the tmRNA gene with the same primer are designated by U, G, C, and A. (n) The lane without any treatment of chemical modification.

been shown in Aquifex aeolicus tmRNA by a chemical and enzymatic footprinting study (Metzinger et al. 2005), although it has not yet been detected in E. coli tmRNA by studies of cross-linking or probing with lead(II) (Barends et al. 2001; Wower et al. 2002; Ivanova et al. 2007). Protection was also found at $G_{14}, U_{16}, U_{17}$, and $G_{19}$ in TLD. No other significant change in the extent of chemical modification by the addition of $\mathrm{SmpB}$ was detected in the region connecting TLD with the mRNA domain.

Chemical modification of tmRNA mutants A84U/U85G, $\mathrm{A} 84 \mathrm{U}, \mathrm{A} 86 \mathrm{C}$, and $\mathrm{A} 86 \mathrm{U}$ was also performed. In the A84U/ U85G mutant, the band intensity of KE modification at 85 was mildly decreased by the addition of SmpB, but CMCT modification at 84 was more significantly decreased (Fig. 2). This is in contrast to DMS modification at 84 in the wild type, in which almost no change in band intensity by the addition of SmpB was detected. In the A84U mutant, a decrease in band intensity by the addition of SmpB was observed predominantly at 85 and almost not or only slightly observed at 84 . In the A86C mutant, the band pattern of chemical modification even in the absence of $\mathrm{SmpB}$ was considerably different from that expected from its sequence, suggesting that the A86C mutation significantly affected the conformation of the upstream region of tmRNA. No significant difference between the chemical modification around 85 in the presence of $S \mathrm{mpB}$ and that in the absence of SmpB was observed. In the A86U mutant, decrease in band intensity by $\mathrm{SmpB}$ was detected not only at 85 but also at 86 (Fig. 2).

\section{Effects of SmpB on chemical modification at $U_{85}$ in trans-translation-defective tmRNA mutants}

The effect of SmpB on chemical modification at $\mathrm{U}_{85}$ was examined in two tmRNA mutants defective in transtranslation, G19C (Hanawa-Suetsugu et al. 2002) and pk1L, in which PK1 is substituted by a single-stranded RNA (Nameki et al. 2000). Almost no or only a faint decrease in chemical modification at $U_{85}$ with increase in $S m p B$ was observed in G19C (Fig. 3), suggesting that the interaction between $\mathrm{U}_{85}$ and $\mathrm{SmpB}$ was dependent on that between TLD and SmpB. Note that the G19C mutation damages the association between tmRNA and $\mathrm{SmpB}$ in a glycerol density gradient centrifugation (Hanawa-Suetsugu et al. 2002). Thus, SmpB is thought to interact both with TLD and with the upstream region. Almost no decrease in chemical modification at $\mathrm{U}_{85}$ with increase in $\mathrm{SmpB}$ was also

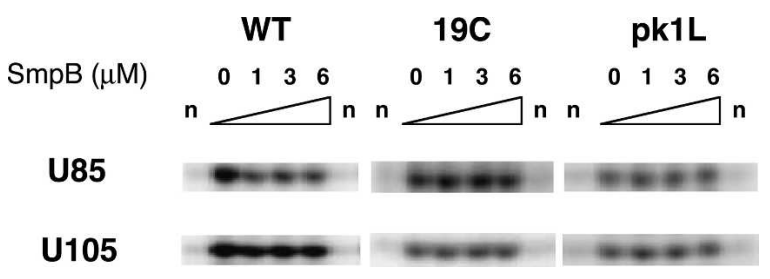

FIGURE 3. Effects of SmpB on chemical modification at $\mathrm{U}_{85}$ in tmRNA mutants defective in trans-translation. Wild-type tmRNA, G19C, and pk1L were modified with CMCT in the presence of $0,1,3$, and $6 \mu \mathrm{M}$ SmpB. The bands at $\mathrm{U}_{85}$ and $\mathrm{U}_{105}$ (control) are shown. (n) The lane without any treatment of chemical modification. 
observed in pk1L (Fig. 3). Unlike G19C, pk1L retains the capacity to associate with $\mathrm{SmpB}$ in a glycerol density gradient (Hanawa-Suetsugu et al. 2002). Perhaps the linearization of PK1, 7 nt upstream of $\mathrm{U}_{85}$ (Fig. 1C), may significantly affect the interaction between $\mathrm{SmpB}$ and the upstream region when maintaining that interaction between SmpB and TLD.

\section{No involvement of the C-terminal tail of SmpB in the interaction with the upstream region of the tag-encoding sequence of tmRNA}

It has been demonstrated that the C-terminal tail of SmpB is required for trans-translation in vivo but not for binding to tmRNA and promoting its association with the ribosome (Jacob et al. 2005; Sundermeier et al. 2005). We prepared a mutant $\mathrm{SmpB}$ with a truncation of $\mathrm{C}$-terminal residues from 133 to 160 ( $\Delta 133$ to 160$)$. As expected, this tailtruncated SmpB failed to promote peptidyl transfer to tmRNA and translation of the resume codon (Fig. 4A,B).

(A)

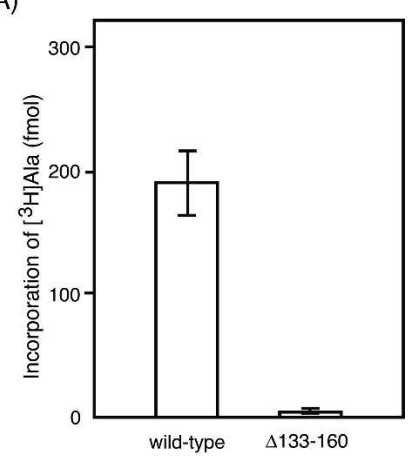

(B)

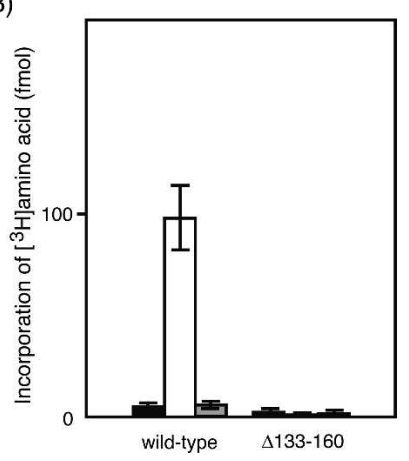

FIGURE 4. Effect of truncation of the C-terminal tail of SmpB on the first and second steps of trans-translation. (A) The level of incorporation of $\left[{ }^{3} \mathrm{H}\right]$ alanine into the polypeptide fraction after 5 min of incubation was monitored to evaluate the first step of transtranslation mediated by tmRNA and wild-type SmpB or C-terminal tail-truncated SmpB $(\Delta 133-460)$. (B) The levels of incorporation of $\left[{ }^{3} \mathrm{H}\right]$ arginine (black), $\left[{ }^{3} \mathrm{H}\right]$ alanine (white), and $\left[{ }^{3} \mathrm{H}\right]$ glutamine (gray) into the polypeptide fraction after $5 \mathrm{~min}$ of incubation were monitored to evaluate the efficiency and fidelity of the second step of trans-translation mediated by tmRNA and SmpB variants.
Using this C-terminal tail-truncated $\mathrm{SmpB}$, chemical modification was performed. $\mathrm{U}_{85}$ in wild-type tmRNA was protected from chemical modification by C-terminal tail-truncated $\mathrm{SmpB}$ as well as by wild-type $\mathrm{SmpB}$, although to different extents (Fig. 5A). In the A84U/ U85G mutant, the pattern of protection by C-terminal tail-truncated $\mathrm{SmpB}$ was also similar to that by wild-type SmpB (Fig. 5B). These results indicate that the protection at the upstream region of the tag-encoding sequence is not due to interaction with the C-terminal tail of SmpB.

\section{DISCUSSION}

In the present study, we established an E. coli in vitro system to evaluate two different steps in the early stage of trans-translation, peptidyl transfer from peptidyl-tRNA to alanyl-tmRNA (the first step), and translation of the resume codon on tmRNA for the tag-peptide (the second step). These two steps can be assessed in a Thermus thermophilus in vitro system recently established in our laboratory (Takada et al. 2007). Using this system, we demonstrated that mutations in the region upstream of the tag-encoding sequence did not affect peptidyl transfer to alanyl-tmRNA, but that several mutations affected the efficiency and/or fidelity of translation of the resume codon. We also found an interaction between the main body of SmpB and the upstream region of the resume codon on tmRNA using chemical footprinting with DMS, KE, and CMCT. We further found that A84U/U85G and A86U mutations shifted both the position of initiation of tag-translation and the position of protection by $\operatorname{SmpB}$ by -1 and +1 , respectively, as summarized in Table 1. This emphasizes the functional significance of the interaction between tmRNA and SmpB presented here.

Metzinger et al. (2005) have shown that more than one molecule of $\mathrm{SmpB}$ from A. aeolicus binds to a tmRNA using surface plasmon resonance. The present study demonstrated that the interaction between the upstream region and SmpB depended on that between TLD and SmpB, although the total number of E. coli SmpB binding to one tmRNA is yet to be determined. Note that no intramolecular tertiary interactions between the tRNA and mRNA domains have been reported. In the span between PK1 and the tag-encoding region, the change in band intensity by the addition of $\mathrm{SmpB}$ was limited to $\mathrm{U}_{85}$. These considerations lead to the idea that the main body of SmpB bridges two separate domains of tmRNA. An alternative possibility that the observed protection of $\mathrm{U}_{85}$ by $\mathrm{SmpB}$ was due to a structural rearrangement around $\mathrm{U}_{85}$ by binding of $\mathrm{SmpB}$ to another portion in tmRNA rather than the direct interaction is less likely. The direct interaction is supported by almost complete protection of the chemical modification of $U_{85}$ by $S m p B$ and by the absence of concomitant change in band intensity around $\mathrm{U}_{85}$. 
(A)

(B)

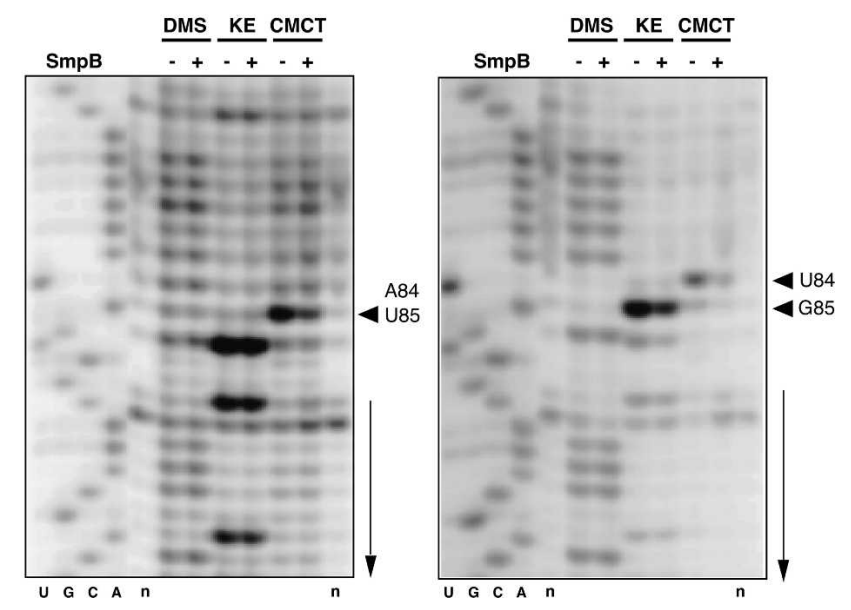

FIGURE 5. Chemical footprinting of tmRNA by C-terminal tailtruncated SmpB. Wild-type $(A)$ tmRNA and $(B)$ A84U/U85G were modified with DMS, KE, and CMCT in the presence and absence of $6 \mu$ M C-terminal tail-truncated SmpB $(\Delta 133-160)$. (Arrowheads) The bands of interest; (arrow) the tag-encoding region with the direction. The lanes of DNA sequencing of pGEMEX-2 carrying the tmRNA gene with the same primer are designated by U, G, C, and A. (n) The lane without any treatment of chemical modification.

The results of chemical probing suggested that the A86C mutation significantly altered the conformation around the upstream region. The A86C mutation also affected the second step of trans-translation but not the first step. This is consistent with the results of a previous study showing that this mutation seriously affects trans-translation in vitro but not binding of tmRNA to the ribosome (Lee et al. 2001). Thus, the conformation around this region is crucial for setting the initial codon for the tag-peptide to the decoding region rather than the processes from the initial binding of tmRNA to the stalled ribosome until peptidyl transfer.

In a crystal structure of $S m p B$ in complex with a fragment of the tRNA-like domain (TLD) from A. aeolicus or T. thermophilus (Gutmann et al. 2003; Bessho et al. 2007), SmpB binds the elbow of TLD, in agreement with the results of biochemical studies (Hanawa-Suetsugu et al. 2002; Nameki et al. 2005). In the complex of SmpB and the TLD fragment, the TLD forms an angle of $120^{\circ}$, and instead SmpB and the TLD form an angle of $90^{\circ}$ as well as canonical tRNAs. When TLD fits the acceptor stem of tRNA toward the peptidyl transferase center on the 50S subunit, the C-terminal tail of $\mathrm{SmpB}$ is thought to extend toward the decoding center on the $30 \mathrm{~S}$ subunit (Gutmann et al. 2003). A chemical footprinting study has also shown that $\mathrm{SmpB}$ interacts with the tRNA-binding sites on the small subunit as well as on the large subunit of the ribosome (Ivanova et al. 2005), which is consistent with a recent cryoelectron microscopic study of complexes of T. thermophilus SmpB and ribosome with or without
alanyl-tmRNA and EF-Tu (Gillet et al. 2006; Kaur et al. 2006). Our recent study of directed hydroxyl radical probing shows that the C-terminal tail of SmpB interacts with the decoding center and the mRNA path of the ribosome (D. Kurita, R. Sasaki, A. Muto, and H. Himeno, unpubl.). Thus, the $\mathrm{C}$-terminal tail may have a role in the interaction with the ribosome rather than tmRNA during trans-translation. This is consistent with a previous finding that a truncation of the C-terminal tail only slightly reduces the affinity with tmRNA (Jacob et al. 2005). The site of this interaction on SmpB has yet to be determined. In complexes of T. thermophilus SmpB, ribosome, alanyl-tmRNA, and EF$\mathrm{Tu}$ (Kaur et al. 2006), the main body of SmpB is relatively near the tag-initiation point.

Taken together, we can draw a model of the early processes of trans-translation. The initial step until the peptidyl transfer from peptidyl-tRNA to alanyl-tmRNA requires the interaction between the C-terminal tail of SmpB and the ribosome rather than that between the main body of SmpB and the upstream region of tmRNA. The next step after the peptidyl-transfer requires the interaction between the main body of SmpB and the upstream region of tmRNA for setting the initial codon for the tag-peptide to the decoding region. It has recently been reported that SmpB can bind to the ribosome even in the absence of tmRNA (Hallier et al. 2004, 2006), and that a tmRNA with two SmpB molecules binds to the ribosome prior to peptidyl transfer (Kaur et al. 2006). These indicate the possibility of an alternative pathway of the preaccommodation step of trans-translation, which does not contradict our model.

The interactions of SmpB with tmRNA can now structurally and functionally be divided into two categories, one with TLD to help the functions as tRNA such as processing, aminoacylation, binding to the ribosome, and

TABLE 1. Protection of the base at 84,85 , or 86 by SmpB and incorporations of amino acids in the second step

\begin{tabular}{lcccccccc}
\hline & \multicolumn{3}{c}{ Band intensity $^{\mathrm{a}}$} & & & \multicolumn{3}{c}{$\begin{array}{c}\text { Incorporation of } \\
\text { amino acid }\end{array}$} \\
\cline { 2 - 3 } \cline { 8 - 9 } & 84 & 85 & 86 & & Arg & Ala & Gln \\
\hline Wild type & 1.00 & 0.71 & 1.00 & & ND $^{\mathrm{c}}$ & 1 & ND \\
A86U & 0.94 & 0.73 & 0.98 & & ND & 0.9 & ND \\
A84U/U85G & 0.61 & 0.60 & 1.01 & & 0.42 & 0.79 & ND \\
A86C & 0.97 & 0.96 & 0.97 & & ND & ND & ND \\
A86U & 1.12 & 0.53 & 0.47 & & ND & 0.45 & 0.19 \\
\hline
\end{tabular}

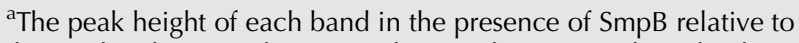
that in the absence of SmpB is shown. The mean value of at least two independent experiments is shown.

${ }^{\mathrm{b}}$ Incorporation of amino acid in the second step of trans-translation mediated by tmRNA variant relative to alanine incorporation in the second step of trans-translation mediated by wild-type tmRNA is shown. The mean value of at least two independent experiments is shown.

${ }^{\mathrm{C}} \mathrm{ND}$ indicates the value $<0.1$. 
peptidyl-transfer, and the other with the upstream region to help the function as mRNA. The latter interaction might be reinforced by the PK1 pseudoknot structure either directly or indirectly, as suggested by the results shown in Figure 3. Because of the functional requirement of a particular distance between the TLD and the tag-encoding region of tmRNA on the ribosome, there should be a structural link between them other than that via PK1, although no intramolecular noncovalent link has been found. The missing link can now be addressed by the present finding that the two separate domains are noncovalently bridged by $\mathrm{SmpB}$.

The present results indicate that the interaction between SmpB and the upstream region of tmRNA is crucial for the precise determination of the initiation point of tag-translation from the first GCA codon. Its modulation apparently caused fluctuation of the initiation point. It has been shown that the 4,5- or 4,6-disubstituted class of aminoglycoside also induces a shift of the initiation point by -1 probably due to binding the decoding center to modulate its conformation (Takahashi et al. 2003; Konno et al. 2004), suggesting the importance of the interaction of the decoding center with any portion on SmpB or tmRNA for precise tag-translation. Thus, two different kinds of interactions might contribute to determination of the initiation point of tag-translation.

The present study provides a new insight into the molecular mechanism of trans-translation. Determination of the interactions of SmpB with tmRNA and the ribosome as well as their rearrangements during the course of transtranslation will lead to further clarification of the molecular mechanism of trans-translation.

\section{MATERIALS AND METHODS}

\section{Preparation of tmRNA and its mutant}

E. coli tmRNA and its mutants were induced from each overproducing strain by the addition of $1.0 \mathrm{mM}$ isopropyl- $\beta-\mathrm{D}$ thiogalactopyranoside and were purified as described previously (Ushida et al. 1994). The nucleic acid fraction was extracted with phenol from the cells and then subjected to ethanol precipitation. The resulting fraction was subjected to differential isopropylalcohol precipitations to roughly remove DNA, followed by incubation with RNase-free DNase I (Pharmacia). tmRNA was purified by electrophoresis on a $5 \%$ polyacrylamide gel containing $7 \mathrm{M}$ urea, and it was then heated for $3 \mathrm{~min}$ at $75^{\circ} \mathrm{C}$ and slowly cooled down for $1 \mathrm{~h}$ at room temperature in the refolding buffer containing $10 \mathrm{mM}$ HEPES-KOH ( $\mathrm{pH}$ 7.5), $5 \mathrm{mM}$ magnesium chloride, and $20 \mathrm{mM}$ ammonium chloride. Spectrophotometric measurements were made to determine the concentration of RNA. Two $A_{260}$ units of tmRNA are usually yielded from $1 \mathrm{~L}$ of culture.

\section{Preparation of total tRNA}

tRNA was prepared from E. coli W3110 $\Delta$ ssrA. The nucleic acid fraction was extracted with phenol and then subjected to ethanol precipitation. After performing phenol extraction and ethanol precipitation, the resulting fraction was subjected to isopropylalcohol precipitations to roughly remove DNA, followed by incubation with RNase-free DNase I (Pharmacia). Total tRNA was fractionated by electrophoresis on a $12 \%$ polyacrylamide gel containing $7 \mathrm{M}$ urea.

\section{Preparation of SmpB and its mutant}

pGEMEX-2 (Promega) harboring the gene for an N-terminally His $_{6}$-tagged SmpB mutant $(\Delta 133-160)$ was prepared from that for $\mathrm{N}$-terminally $\mathrm{His}_{6}$-tagged SmpB (Hanawa-Suetsugu et al. 2002). C-terminally $\mathrm{His}_{6}$-tagged SmpB (Hanawa-Suetsugu et al. 2002) or N-terminally $\mathrm{His}_{6}$-tagged SmpB mutant $(\Delta 133-160)$ was induced from each overproducing strain by the addition of $1.0 \mathrm{mM}$ IPTG and was purified as described previously (Hanawa-Suetsugu et al. 2002).

\section{In vitro analysis of peptidyl transfer to alanyl-tmRNA and translation of the resume codon on tmRNA}

The translation factors and the stalled ribosome were prepared according to Asano et al. (2005) E. coli tRNA $^{\text {Phe }}$ (Sigma) was aminoacylated with $\left[{ }^{14} \mathrm{C}\right]$ phenylalanine by $E$. coli phenylalanyltRNA synthetase; $80 \%-90 \%$ of tRNA ${ }^{\text {Phe }}$ was aminoacylated. The translation reaction mixture $(50 \mu \mathrm{L})$ contained 10 pmol of ribosome, $100 \mathrm{pmol}$ of (UUC) ${ }_{10}$ as an mRNA, 100 pmol of EF-Tu, 3 pmol of EF-G, 10 pmol of $\left[{ }^{14} \mathrm{C}\right]$ phenylalanyl-tRNA ${ }^{\text {Phe }}, 2 \mathrm{mM}$ spermidine, $1 \mathrm{mM}$ ATP, $0.2 \mathrm{mM} \mathrm{GTP}, 80 \mathrm{mM}$ Tris- $\mathrm{HCl}$ (pH 7.5), $30 \mathrm{mM}$ ammonium chloride, $5 \mathrm{mM}$ magnesium acetate, and 2.5 $\mathrm{mM}$ dithiothreitol.

tmRNA was aminoacylated with $\left[{ }^{3} \mathrm{H}\right]$ alanine by alanyl-tRNA synthetase from E. coli (Tamura et al. 1991). After the translation reaction mixture had been incubated for $15 \mathrm{~min}$ at $37^{\circ} \mathrm{C}, 5 \mathrm{pmol}$ of $\left[{ }^{3} \mathrm{H}\right]$ alanyl-tmRNA, and 30 pmol of $\mathrm{SmpB}$ was added. After different periods of incubation at $37^{\circ} \mathrm{C}$, an aliquot of the mixture was withdrawn and put in $5 \mathrm{~mL}$ of hot $5 \%$ trichloroacetic acid to stop the reaction, and the radioactivity of the acid-insoluble fraction was measured by using a liquid scintillation counter (LSC-3500; Aloka).

Total tRNA from E. coli was aminoacylated with $\left[{ }^{3} \mathrm{H}\right]$ alanine, $\left[{ }^{3} \mathrm{H}\right]$ arginine, or $\left[{ }^{3} \mathrm{H}\right]$ glutamine by each aminoacyl-tRNA synthetase prepared from E. coli W3110 by anion exchange column chromatography (DEAE-Toyopearl 650; Tosoh). Incorporation of alanine, arginine, or glutamine into polypeptide was determined by mixing the stalled complex of ribosome with 5 pmol of each aminoacyl-tRNA, 5 pmol of alanyl-tmRNA, and 30 pmol of SmpB. After different periods of incubation at $37^{\circ} \mathrm{C}$, an aliquot of the mixture was withdrawn and put in $5 \mathrm{~mL}$ of hot $5 \%$ trichloroacetic acid to stop the reaction. It was boiled for $10 \mathrm{~min}$, and the radioactivity of the acid-insoluble fraction was measured by using a liquid scintillation counter. The final value of amino acid incorporation was obtained by subtracting the value in the absence of SmpB from that in the presence of SmpB.

\section{Chemical modification}

Chemical modifications and subsequent reverse transcription were carried out according to the method described previously (Takahashi et al. 2003) with a slight modification. After $1 \mu \mathrm{M}$ tmRNA had been incubated for $15 \mathrm{~min}$ at $37^{\circ} \mathrm{C}$ in a $50 \mu \mathrm{L}$ 
reaction mixture of $80 \mathrm{mM}$ K-HEPES ( $\mathrm{pH}$ 7.0) for dimethyl sulfate (DMS), $80 \mathrm{mM} \mathrm{K-HEPES} \mathrm{(pH} \mathrm{7.8),} \mathrm{for} \mathrm{kethoxal} \mathrm{(KE)} \mathrm{or}$ $80 \mathrm{mM}$ K-borate ( $\mathrm{pH}$ 8.0) for 1-cyclohexyl-3-(2-morpholinoethyl) carbodiimide metho- $p$-toluene sulfonate (CMCT). The reaction mixture also contained $10 \mathrm{mM}$ magnesium chloride, $150 \mathrm{mM}$ potassium chloride, $6 \mathrm{mM} 2$-mercaptoethanol, and 0 or $6 \mu \mathrm{M}$ purified SmpB; $5 \mu \mathrm{L}$ of $16.6 \%$ (v/v) DMS in $95 \%$ ethanol, $5 \mu \mathrm{L}$ of $37 \mathrm{mg} / \mathrm{mL} \mathrm{KE}$, or $5 \mu \mathrm{L}$ of $42 \mathrm{mg} / \mathrm{mL}$ CMCT was added, and the mixture was incubated for $10 \mathrm{~min}$ at $37^{\circ} \mathrm{C}$.

The reverse transcriptase reaction was carried out in a $20 \mu \mathrm{L}$ reaction mixture containing $50 \mathrm{mM}$ Tris- $\mathrm{HCl}(\mathrm{pH} 8.3), 75 \mathrm{mM}$ potassium chloride, $3 \mathrm{mM}$ magnesium chloride, $10 \mathrm{mM}$ dithiothreitol, $1 \mathrm{mM}$ each of dNTP, $2.6 \mathrm{pmol}$ of chemically modified tmRNA, 2 pmol of $5^{\prime}$ Texas Red-labeled DNA primer (5'AAGAGAGGUCAAACCCAA- $3^{\prime}$ ) complementary to a portion within PK2, 10 units of ribonuclease inhibitor from human placenta (Takara), and 60 units of reverse transcriptase from Molony murine leukemia virus (RNase H-; Takara). After the addition of $3 \mu \mathrm{L}$ of a stop solution containing $7 \mathrm{M}$ urea and $0.5 \%$ bromophenol blue, the positions of modifications were analyzed using a fluorescence DNA sequencer (Hitachi, SQ-5500).

\section{ACKNOWLEDGMENTS}

We thank the staff of the Gene Research Center of Hirosaki University for the use of the facility. We also thank the 21th COE program of Iwate University. This work was supported by a grantin-aid for scientific research from the Ministry of Education, Science, Sports and Culture, Japan, to A.M. and H.H. (No. 14035201), a grant-in-aid for scientific research from the Japan Society for the Promotion of Science to A.M. and H.H. (No. 17380061), and a Grant for Priority Research Designated by the President of Hirosaki University to H.H. and A.M.

Received April 23, 2007; accepted July 5, 2007.

\section{REFERENCES}

Abo, T., Inada, T., Ogawa, K., and Aiba, H. 2000. SsrA-mediated tagging and proteolysis of LacI and its role in the regulation of lac operon. EMBO J. 19: 3762-3769.

Andersen, E.S., Rosenblad, M.A., Larsen, N., Westergaard, J.C., Burks, J., Wower, I.K., Wower, J., Gorodkin, J., Samuelsson, T., and Zwieb, C. 2006. The tmRDB and SRPDB resources. Nucleic Acids Res. 34: D163-D168.

Asano, K., Kurita, D., Takada, K., Konno, T., Muto, A., and Himeno, H. 2005. Competition between trans-translation and termination or elongation of translation. Nucleic Acids Res. 33: 5544-5552.

Barends, S., Wower, J., and Kraal, B. 2000. Kinetic parameters for tmRNA binding to alanyl-tRNA synthetase and elongation factor $\mathrm{Tu}$ from Escherichia coli. Biochemistry 39: 2652-2658.

Barends, S., Karzai, A.W., Sauer, R.T., Wower, J., and Kraal, B. 2001. Simultaneous and functional binding of SmpB and EF-Tu-GTP to the alanyl-acceptor arm of tmRNA. J. Mol. Biol. 314: 9-21.

Bessho, Y., Shibata, R., Sekine, S., Murayama, K., Higashijima, K., Hori-Takemoto, C., Shirouzu, M., Kuramitsu, S., and Yokoyama, S. 2007. Structural basis for functional mimicry of long-variable-arm tRNA by transfer-messenger RNA. Proc. Natl. Acad. Sci. 104: 8293-8298.
Dong, G., Nowakowski, J., and Hoffman, D.W. 2002. Structure of small protein B: The protein component of the tmRNA-SmpB system for ribosome rescue. EMBO J. 21: 1845-1854.

Felden, B., Himeno, H., Muto, A., McCutcheon, J.P., Atkins, J.F., and Gesteland, R.F. 1997. Probing the structure of the Escherichia coli 10Sa RNA (tmRNA). RNA 3: 89-104.

Fujihara, A., Tomatsu, H., Inagaki, S., Tadaki, T., Ushida, C., Himeno, H., and Muto, A. 2002. Detection of tmRNA-mediated trans-translation products in Bacillus subtilis. Genes Cells 7: 343-350.

Gillet, R., Kaur, S., Li, W., Hallier, M., Felden, B., and Frank, J. 2006. Scaffolding as an organizing principle in trans-translation: The roles of small protein B and ribosomal protein S1. J. Biol. Chem. 282: 6356-6363.

Gueneau de Novoa, P. and Williams, K.P. 2004. The tmRNA website: Reductive evolution of tmRNA in plastids and other endosymbionts. Nucleic Acids Res. 32: D104-D108.

Gutmann, S., Haebel, P.W., Metzinger, L., Sutter, M., Felden, B., and Ban, N. 2003. Crystal structure of the transfer-RNA domain of transfer-messenger RNA in complex with SmpB. Nature 424: 5503-5509.

Hallier, M., Ivanova, N., Rametti, A., Pavlov, M., Ehrenberg, M., and Felden, B. 2004. Pre-binding of small protein B to a stalled ribosome triggers trans-translation. J. Biol. Chem. 279: 2597825985.

Hallier, M., Desreac, J., and Felden, B. 2006. Small protein B interacts with the large and the small subunits of a stalled ribosome during trans-translation. Nucleic Acids Res. 34: 1935-1943.

Hanawa-Suetsugu, K., Bordeau, V., Himeno, H., Muto, A., and Felden, B. 2001. Importance of the conserved nucleotides around the tRNA-like structure of Escherichia coli transfer-messenger RNA for protein tagging. Nucleic Acids Res. 29: 4663-4673.

Hanawa-Suetsugu, K., Takagi, M., Inokuchi, H., Himeno, H., and Muto, A. 2002. SmpB functions in various steps of transtranslation. Nucleic Acids Res. 30: 1620-1629.

Himeno, H., Sato, M., Tadaki, T., Fukushima, M., Ushida, C., and Muto, A. 1997. In vitro trans translation mediated by alaninecharged 10Sa RNA. J. Mol. Biol. 268: 803-808.

Hong, S.J., Tran, Q.A., and Keiler, K.C. 2005. Cell cycle-regulated degradation of tmRNA is controlled by RNase R and SmpB. Mol. Microbiol. 57: 565-575.

Huang, C., Wolfgang, M.C., Withey, J., Koomey, M., and Friedman, D.I. 2000. Charged tmRNA but not tmRNA-mediated proteolysis is essential for Neisseria gonorrhoeae viability. EMBO J. 19: 1098-1107.

Ivanova, N., Pavlov, M.Y., Bouakaz, E., Ehrenberg, M., and Schiavone, L.H. 2005. Mapping the interaction of SmpB with ribosomes by footprinting of ribosomal RNA. Nucleic Acids Res. 33: 3529-3539.

Ivanova, N., Lindell, M., Pavlov, M., Holmberg Schiavone, L., Wagner, E.G., and Ehrenberg, M. 2007. Structure probing of tmRNA in distinct stages of trans-translation. RNA 13: 713-722.

Jacob, Y., Sharkady, S.M., Bhardwaj, K., Sanda, A.A., and Williams, K.P. 2005. Function of the SmpB tail in transfermessenger RNA translation revealed by a nucleus-encoded form. J. Biol. Chem. 280: 5503-5509.

Karzai, A.W., Susskind, M.M., and Sauer, R.T. 1999. SmpB, a unique RNA-binding protein essential for the peptide-tagging activity of SsrA (tmRNA). EMBO J. 18: 3793-3799.

Kaur, S., Gillet, R., Li, W., Gursky, R., and Frank, J. 2006. Cryo-EM visualization of transfer messenger RNA with two SmpBs in a stalled ribosome. Proc. Natl. Acad. Sci. 103: 16484-16489.

Keiler, K.C. and Shapiro, L. 2003. tmRNA in Caulobacter crescentus is cell cycle regulated by temporally controlled transcription and RNA degradation. J. Bacteriol. 185: 1825-1830.

Keiler, K.C., Waller, P.R.H., and Sauer, R.T. 1996. Role of a peptide tagging system in degradation of protein synthesized from messenger RNA. Science 271: 990-993.

Komine, Y., Kitabatake, M., Yokogawa, T., Nishikawa, K., and Inokuchi, H. 1994. A tRNA-like structure is present in 10Sa 
RNA, a small stable RNA from Escherichia coli. Proc. Natl. Acad. Sci. 91: 9223-9227.

Konno, T., Takahashi, T., Kurita, D., Muto, A., and Himeno, H. 2004. A minimum structure of aminoglycosides that causes an initiation shift of trans-translation. Nucleic Acids Res. 32: 4119-4126.

Lee, S., Ishii, M., Tadaki, T., Muto, A., and Himeno, H. 2001. Determinants on tmRNA for initiating efficient and precise transtranslation: Some mutations upstream of the tag-encoding sequence of Escherichia coli tmRNA shift the initiation point of trans-translation in vitro. RNA 7: 999-1012.

Metzinger, L., Hallier, M., and Felden, B. 2005. Independent binding sites of small protein B onto transfer-messenger RNA during trans-translation. Nucleic Acids Res. 33: 2384-2394.

Muto, A., Ushida, C., and Himeno, H. 1998. A bacterial RNA that functions as both a tRNA and an mRNA. Trends Biochem. Sci. 23: 25-29.

Muto, A., Fujihara, A., Ito, K., Matsuno, J., Ushida, C., and Himeno, H. 2000. Requirement of transfer-messenger RNA (tmRNA) for the growth of Bacillus subtilis under stresses. Genes Cells 5: 627-636.

Nameki, N., Felden, B., Atkins, J.F., Gesteland, R.F., Himeno, H., and Muto, A. 1999a. Functional and structural analysis of a pseudoknot upstream of the tag-encoded sequence in E. coli tmRNA. J. Mol. Biol. 286: 733-744.

Nameki, N., Chattopadhyay, P., Himeno, H., Muto, A., and Kawai, G. 1999b. An NMR and mutational analysis of an RNA pseudoknot of E. coli tmRNA involved in trans-translation. Nucleic Acids Res. 27: 3667-3675.

Nameki, N., Tadaki, T., Himeno, H., and Muto, A. 2000. Three of four pseudoknots in tmRNA are interchangeable and are substitutable with single-stranded RNAs. FEBS Lett. 470: 345-349.

Nameki, N., Someya, T., Okano, S., Suemasa, R., Kimoto, M., Hanawa-Suetsugu, K., Terada, T., Shirouzu, M., Hirao, I., Takaku, H., et al. 2005. Interaction analysis between tmRNA and SmpB from Thermus thermophilus. J. Biochem. 138: 729-739.

Rudinger-Thirion, J., Giegé, R., and Felden, B. 1999. Aminoacylated tmRNA from Escherichia coli interacts with procaryotic elongation factor Tu. RNA 5: 989-992.

Shimizu, Y. and Ueda, T. 2002. The role of SmpB protein in transtranslation. FEBS Lett. 514: 74-77.
Someya, T., Nameki, N., Hosoi, H., Suzuki, S., Hatanaka, H., Fujii, M., Terada, T., Shirouzu, M., Inoue, Y., Shibata, T., et al. 2003. Solution structure of a tmRNA-binding protein, $\mathrm{SmpB}$, from Thermus thermophilus. FEBS Lett. 535: 94-100.

Stepanov, V.G. and Nyborg, J. 2003. tmRNA from Thermus thermophilus. Interaction with alanyl-tRNA synthetase and elongation factor Tu. Eur. J. Biochem. 270: 463-475.

Sundermeier, T.R., Dulebohn, D.P., Cho, H.J., and Karzai, A.W. 2005. A previously uncharacterized role for small protein B (SmpB) in transfer messenger RNA-mediated trans-translation. Proc. Natl. Acad. Sci. 102: 2316-2321.

Takada, K., Takemoto, C., Kawazoe, M., Konno, T., HanawaSuetsugu, K., Lee, S., Shirouzu, M., Yokoyama, S., Muto, A., and Himeno, H. 2007. In vitro trans-translation of Thermus thermophilus: Ribosomal protein S1 is not required for the early stage of trans-translation. RNA 13: 503-510.

Takahashi, T., Konno, T., Muto, A., and Himeno, H. 2003. Various effects of paromomycin on tmRNA-directed trans-translation. J. Biol. Chem. 278: 27672-27680.

Tamura, K., Asahara, H., Himeno, H., Hasegawa, T., and Shimizu, M. 1991. Identity elements of E. coli tRNA ${ }^{\mathrm{Ala}}$. J. Mol. Recognit. 4: 129132.

Ushida, C., Himeno, H., Watanabe, T., and Muto, A. 1994. tRNA-like structures in 10Sa RNAs of Mycoplasma capricolum and Bacillus subtilis. Nucleic Acids Res. 22: 3392-3396.

Williams, K.P. and Bartel, D.P. 1996. Phylogenetic analysis of tmRNA secondary structures. RNA 2: 1306-1310.

Williams, K.P., Martindale, K.A., and Bartel, D.P. 1999. Resuming translation on tmRNA: A unique mode of determining a reading frame. $E M B O$ J. 18: 5423-5433.

Withey, J.H. and Friedman, D.I. 2003. A salvage pathway for protein structures: tmRNA and trans-translation. Annu. Rev. Microbiol. 56: 1062-1077.

Wower, J., Zwieb, C., Guven, S.A., and Wower, I. 2000. Binding and cross-linking of tmRNA to ribosomal protein S1, on and off the Escherichia coli ribosome. EMBO J. 19: 6612-6621.

Wower, J., Zwieb, C.W., Hoffman, D.W., and Wower, I.K. 2002. SmpB: A protein that binds to double stranded segments in tmRNA and tRNA. Biochemistry 41: 8826-8836.

Wower, I.K., Zwieb, C., and Wower, J. 2004. Contributions of pseudoknots and protein $\mathrm{SmpB}$ to the structure and function of tmRNA in trans-translation. J. Biol. Chem. 279: 54202-54209. 

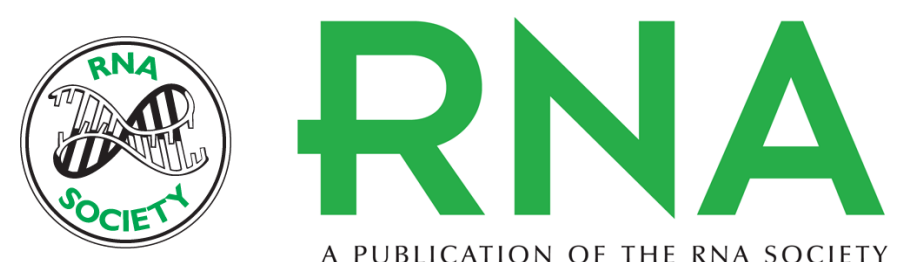

A PUBLICATION OF THE RNA SOCIETY

\section{A functional interaction of SmpB with tmRNA for determination of the resuming point of trans-translation}

Takayuki Konno, Daisuke Kurita, Kazuma Takada, et al.

RNA 2007 13: 1723-1731 originally published online August 13, 2007

Access the most recent version at doi:10.1261/rna.604907

References This article cites 51 articles, 21 of which can be accessed free at:

http://rnajournal.cshlp.org/content/13/10/1723.full.html\#ref-list-1

License

Email Alerting Receive free email alerts when new articles cite this article - sign up in the box at the

Service top right corner of the article or click here. 